\title{
A simulation-based actuator surface parameterization for large-eddy simulation of propeller wakes
}

\author{
Fei Liao $^{\text {a }}$, Shizhao Wang ${ }^{\text {a,b }}$, Xiaolei Yang ${ }^{\text {a,b, }}{ }^{*}$, Guowei He ${ }^{\text {a,b }}$ \\ ${ }^{a}$ State Key Laboratory of Nonlinear Mechanics, Institute of Mechanics, Chinese Academy of Sciences, Beijing, 100190, China \\ ${ }^{\mathrm{b}}$ School of Engineering Sciences, University of Chinese Academy of Sciences, Beijing, 100049, China
}

\section{A R T I C L E I N F O}

\section{Keywords:}

Actuator surface

Simulation-based parameterization

Propeller

LES

RANS

\begin{abstract}
A B S T R A C T
The existing actuator surface models for wind turbines, which are developed based on the blade element method using the two-dimensional assumption, cannot be easily applied to some marine propellers of highly threedimensional geometry. In the present paper, a simulation-based actuator surface parameterization for largeeddy simulation (LES) of propeller wakes with the distributed forces computed using a precursor Reynoldsaveraged Navier-Stokes (RANS) simulation is proposed to address this issue. Three different actuator surface models, which apply the RANS computed forces directly on the blade surface and apply the RANS-computed forces with/without averaging in the chordwise direction on the 2-D elements of the thickless actuator surface, respectively, are tested. The mean velocity profiles at downstream locations of the propeller are found to be similar for the three different models. The turbulence kinetic energy (TKE) profiles computed from the three different models, on the other hand, are found to be very different in terms of the location and size of the high TKE region. This study indicates the importance of properly modeling the geometrical and hydrodynamic effects of blade in actuator surface models in order to accurately predict propeller wakes.
\end{abstract}

\section{Introduction}

The turbulent flow around a propeller in real-world conditions is featured by high Reynolds number, complex geometric and moving boundaries. Direct numerical simulation (DNS) of such complex turbulent flows remains a great challenge even with the next generation exascale computing capability. Turbulence models of different fidelity, such as large-eddy simulation (LES) and Reynolds-averaged NavierStokes (RANS) method, exist for turbulent flow simulations. LES, which is more accurate than RANS in predicting the unsteadiness of turbulent flows, while requires significantly lower computational cost compared with DNS, has been widely used in the simulation of turbulent flows around wind turbines and propellers (Wu and Porté-Agel, 2011; Yang et al., 2015; Kumar and Mahesh, 2017; Kang et al., 2012, 2014; Elias et al., 2015; Posa and Balaras, 2018; Posa et al., 2019a). The thickness of a blade is several orders of magnitude smaller than the diameter of the propeller. This poses significant constraints on the spatial and temporal resolutions required for simulating such flows. Simulations directly resolving the blade geometry have been carried out in the literature. For instance, large-eddy simulations of an axial-flow hydrokinetic turbine were carried out in (Kang et al., 2012, 2014) using curvilinear immersed boundary method. In (Elias et al., 2015; Posa et al., 2019b) and (Posa et al., 2015) large-eddy simulations of submarine propellers and mixed-flow pump were carried out, respectively. Recently, 1.7 billion nodes have been used to analyze the wake feature of a propeller in presence of an upstream rudder by Posa et al. (2019a). These geometry-resolving simulations can predict the coherent flow structures at the blade chord scale but at a very high computational cost. To reduce the computational cost, different actuator type models have been developed in the literature (Froude, 1889; Ganesh Rajagopalan et al., 1990; Dobrev et al., 2007). The accuracy of the existing actuator type models, which are implemented based on the blade element approach, depends on the quality of the force coefficients (which may not even exist for some propeller blades) as well as the corrections for the three-dimensional effect. Furthermore, the propeller blade cannot be easily divided to two-dimensional elements because it's highly three-dimensional geometry. In the present work, we develop a RANS-based actuator surface model, which does not depend on three-dimensional corrections and can be applied to blades of arbitrarily three-dimensional geometries, and apply the developed model to a propeller.

In actuator type models, the impact of turbine or propeller on

\footnotetext{
* Corresponding author. State Key Laboratory of Nonlinear Mechanics, Institute of Mechanics, Chinese Academy of Sciences, Beijing, 100190, China.

E-mail address: xyang@imech.ac.cn (X. Yang).
} 


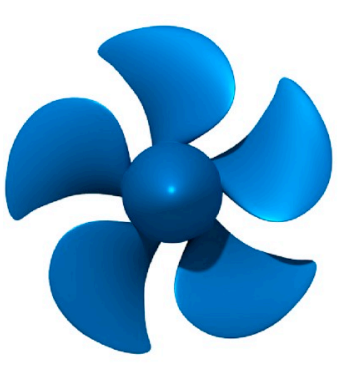

(a) Front view

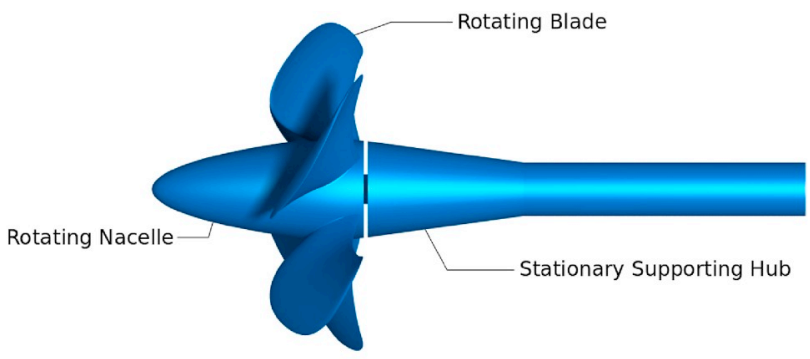

(b) Side view

Fig. 1. Geometry of the VP1304 Propeller. The flow in (b) is from left to right.

incoming flows is taken into account by distributed forces, which are added to the Navier-Stokes equations as source terms. No need to directly resolve the geometry of turbine or propeller and the boundary layer around the blade leads to simple computational meshes and significantly reduces the computational cost. How to construct the distributed forces, which determines the accuracy of the flow solver, is vital to the success of actuator type models. There are three different types of actuator models employed in wind turbine simulations, i.e. the actuator disk model (ADM), the actuator line model (ALM) and the actuator surface model (ASM). The ADM (Froude, 1889; Yang et al., 2012) uses a circular permeable disk to represent the rotating blades. The distributed forces on the permeable disk are computed based on the one-dimensional momentum theory (Martin, 2015) or the blade element momentum method (Froude, 1889). ADM can predict velocity deficits with reasonable accuracy. However, it cannot predict the blade tip vortex since the effect of an individual blade is not taken into account. In the ALM (Ganesh Rajagopalan et al., 1990; Sørensen and Shen, 2002; Shen et al., 2005; Martinez et al., 2016; Yang et al., 2015), on the other hand, each blade is represented by a rotating line with distributed forces, which are determined based on the blade element theory using the geometrical parameters (chord length and twist angle), lift and drag coefficients, and the local inflow information (velocity and angle of attack). Due to the presence of each individual blade as a rotating line, the ALM can capture more flow structures than the ADM could, such as the blade tip vortex. However, it still suffers from some simplifications, such as the line representation of the blade and the use of the lift and drag coefficients from two dimensional foils, which may affect the accuracy of the ALM. In the ASM (Dobrev et al., 2007; Shen et al., 2009; Yang and Sotiropoulos, 2018), each blade is represented using an actuator surface. Due to the refined representation of the blade geometry, the ASM is regarded to be more accurate than the ALM. The distributed forces on the actuator surface are computed in different ways. In the model of Dobrev et al. (2007), the forces are computed using a pressure jump on the mean surface of the blades by combining BEM with Fluent in an iterative way. In Shen et al.'s model (Shen et al., 2009), the ASM of Dobrev et al. was further generalized using a set of predefined functions that depend on angle of attack and airfoil shape with tabulated data or empirical formulations for general turbine flows. In the model of Yang and Sotiropoulos (2018), the nacelle geometry was taken into consideration using the actuator surface concept.

In the early research on marine propellers, the volume force method, which is very similar to the actuator-type model in wind turbine simulations, was used to simplify the complexity introduced by resolving the flow over the propeller. In 1988, the volume force method was adopted by Stern et al. (1988) to simulate the propeller-hull interaction. In 1997, Kawamura et al. (1997) focused on the self-propelling of five tanker models with the volume force method. In 2009, Choi et al. (2009) adopted the volume force method with a RANS method for the prediction of ship-speed performance of VLCC (Very Large Crude Carriers) ship. In the present paper, we focus on developing an actuator surface model for large-eddy simulation of the flow over marine propellers. As will be shown in Fig. 1, the chord length and the propeller radius of a marine propeller are of the same order of magnitude. Furthermore, the marine propeller has large twist angle and pitch angle. This makes the blade element method and the two-dimensional aerodynamic coefficients employed in the existing actuator models inaccurate for the marine propeller simulations. The RANS method, on the other hand, can predict the force distribution on the surface of the propeller of any shape. It has been shown in (Peng et al., 2013; Da-Qing, 2006) that RANS can accurately predict the open water characteristics (e.g., thrust coefficient, torque coefficient and open water efficiency) of a propeller. In this work, we propose a new actuator surface model, which computes the distributed forces using the RANS simulation, which are then employed in the LES of propeller wake simulation. Compared with existing ASM or ALM, the proposed ASM can model the distributed forces with a higher accuracy and can be applied to both turbines and propellers with arbitrary geometries.

This paper is organized as follows. After briefly describing the flow solver in section 2, we present the proposed actuator surface model with three different simplification in sections 3 . In section 4, we then discuss the computed results of a propeller wake. At last, we summarize the present work in section 5 .

\section{Flow solver}

\subsection{Solver for large-eddy simulation on Cartesian grids}

In this section we describe the employed flow solver for large-eddy simulation of propeller wakes. The governing equations are the spatially filtered incompressible Navier-Stokes equations on Cartesian grids, which are shown as follows:

$$
\begin{aligned}
& \frac{\partial \tilde{u}_{j}}{\partial x_{j}}=0, \\
& \frac{\partial \tilde{u}_{i}}{\partial t}+\frac{\tilde{u}_{i} \tilde{u}_{j}}{\partial x_{j}}=-\frac{1}{\rho} \frac{\partial \tilde{p}}{\partial x_{i}}-\frac{\partial \tau_{i j}}{\partial x_{j}}+\frac{1}{\operatorname{Re}} \frac{\partial^{2} \tilde{u}_{i}}{\partial x_{j} \partial x_{j}}+f_{i},
\end{aligned}
$$

where $\boldsymbol{x}=\left(x_{1}, x_{2}, x_{3}\right)=(x, y, z)$ is the Cartesian coordinate, $t$ is time, $\tilde{\boldsymbol{u}}=$ $\left(\tilde{u}_{1}, \tilde{u}_{2}, \tilde{u}_{3}\right)=(\tilde{u}, \tilde{v}, \tilde{w})$ is the filtered velocity, $\tilde{p}$ is the filtered pressure, $\rho$ is the constant density, and $\boldsymbol{f}=\left(f_{1}, f_{2}, f_{3}\right)$ is the source term for distributed force. The Reynolds number is $R e=\frac{U L}{\nu}$, where $U$ is the freestream velocity, $L$ is the reference length and $\nu$ is the kinematic viscosity. The subgrid scale stress $\tau_{i j}=\widetilde{u_{i} u_{j}}-\tilde{u}_{i} \tilde{u}_{j}$ is modeled by the wall-adapting local eddy-viscosity (WALE) model (Nicoud and Ducros, 1999), which is expressed as follows

$$
\nu_{t}=\left(C_{w} \Delta\right)^{2} \frac{\left(\tilde{S}_{i j}^{d} \tilde{S}_{i j}^{d}\right)^{3 / 2}}{\left(\bar{S}_{i j} \bar{S}_{i j}\right)^{5 / 2}+\left(\tilde{S}_{i j}^{d} \tilde{S}_{i j}^{d}\right)^{5 / 4}},
$$

where $\bar{S}_{i j}$ is the strain rate tensor, and $\Delta$ is the subgrid characteristic length scale, which is defined as the cube root of the cell volume. $C_{w}=$ 


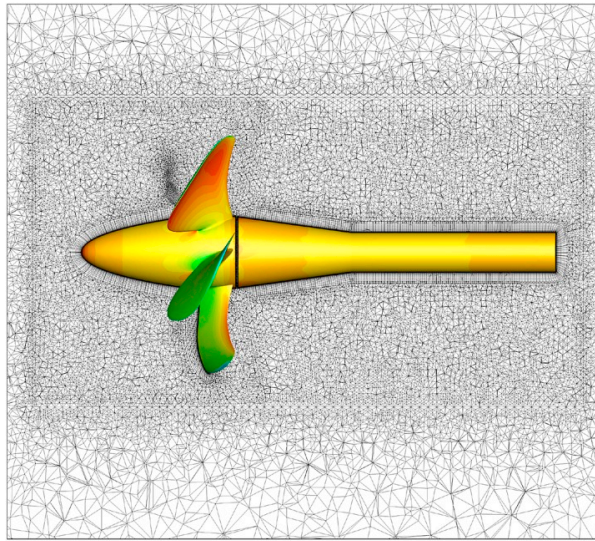

(a) Unstructured mesh for RANS

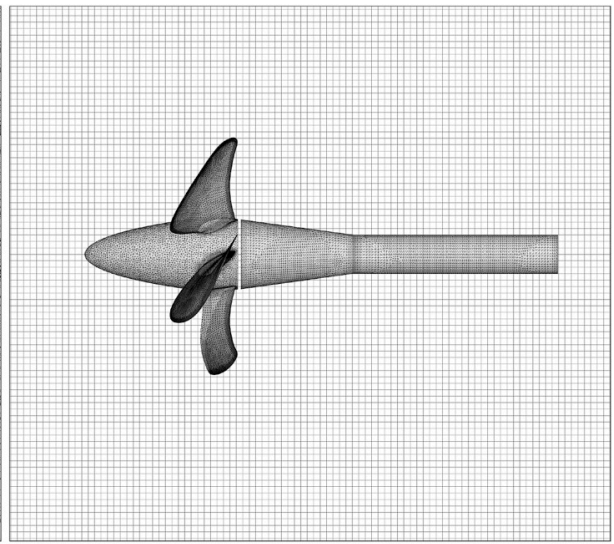

(b) Cartesian grid for LES with unstructured surface element

Fig. 2. Illustration for the meshes using in (a) RANS and (b) LES with the model M1, in which the geometry of the propeller used in LES is the same as the one used in RANS. The RANS computed forces are directly employed in LES with the actuator surface model, M1.

0.8 is used in the following research. And

$\tilde{S}_{i j}^{d}=\frac{1}{2}\left(\tilde{g}_{i j}^{2}+\tilde{g}_{j i}^{2}\right)-\frac{1}{3} \delta_{i j} \tilde{g}_{k k}^{2}$

where $\delta_{i j}$ is the Kronecker delta, $\tilde{g}_{i j}^{2}=\tilde{g}_{i k} \tilde{g}_{k j}$ and $\tilde{g}_{i j}=\partial \tilde{u}_{i} / \partial x_{j}$. The governing equations are solved on the Cartesian Eulerian grid using the finite-difference method. Second-order central difference is used for the spatial discretization, and the second-order Adams-Bashforth method is adopted for the temporal advancement. Velocity and pressure are coupled with the fractional-step method, in which the Poisson equation is solved to satisfy the divergence-free condition. More details about the numerical algorithms can be found in (Wang et al., 2016).

\subsection{Solver for RANS simulation on unstructured body-fitted mesh}

In this paper, RANS simulation is performed with the ANSYS Fluent software using the pressure-based solver. Incompressible simulation is realized through fixed density of water and disabled energy equation. Turbulence is modeled by Menter's two-equation $k$ - $\omega$ SST model (Menter, 1994). Pressure-velocity coupling is achieved using SIMPLE method. Gradient, pressure and momentum are discretized through least squares cell based scheme, 2nd-order central scheme and 2nd-order upwind scheme, respectively. Steady simulation is carried out using unstructured body-fitted mesh with rotating reference frame technique, in which a stationary domain and a rotating domain need to be separately defined in the mesh.

\section{Simulation-based actuator surface model for propeller wakes}

In actuator type models, the effect of the propeller is represented by forcing terms as shown in Eqs. (1) with the actuator surface treated as immersed boundary embedded in Cartesian grids. Different meshes are employed for the actuator type model and the fluid, which makes the forcing term employed for solving the flow cannot be computed directly. To compute the forcing term in Eqs. (1), a force distribution process is carried out as follows:

$\boldsymbol{f}(\boldsymbol{x})=\sum_{\boldsymbol{X} \in G} f(\boldsymbol{X}) \delta_{h}(\boldsymbol{x}-\boldsymbol{X}) \Delta S(\boldsymbol{X})$,

where $\boldsymbol{X}$ and $\boldsymbol{x}$ denote the coordinates of the grid nodes of the actuator model mesh and the fluid mesh, respectively, $G$ denotes the collection of surface mesh cells discretizing the geometry employed in the actuator model, $\Delta S$ is the area of the surface mesh cell at $X$, and $\delta_{h}$ is the discrete delta function for the force distribution the same as that employed in the direct forcing immersed boundary method (Yang et al., 2009), which can guarantee the force and moment conservations during the force distribution. Different actuator type models differentiate in the way how the forcing term $f(X)$ in Eq. (4) is calculated and how the geometry $G$ is defined. In the actuator disk model (Yang et al., 2012), the propeller geometry is represented by a disk with the forcing term calculated based on the one-dimensional momentum theory. In the actuator line model (Yang et al., 2015), the blade is represented by a rotating line with the distributed forces computed based on blade element method using the geometric parameters, the aerodynamic coefficients and the local incoming velocity. In the actuator surface model proposed by Yang and Sotiropoulos (2018) the forces are computed in the same way as in the actuator line model but are distributed from a surface assuming a uniform force distribution in the chordwise direction. In the actuator surface model proposed by Shen et al. (2009), on the other hand, the forces are computed based the pressure coefficients on the surface of the airfoil.

In this work, we develop an actuator surface model for simulating the wake of a marine propeller. Specifically, we considers the VP1304 propeller as shown in Fig. 1, which consists of the rotating blades, the rotating nacelle and the stationary supporting hub. The rotating nacelle and the stationary supporting hub are modeled using the actuator surface model for nacelle proposed in (Yang and Sotiropoulos, 2018), in which the normal force and the tangential force on the surface are computed by satisfying the non-penetration boundary condition and by specifying the friction coefficient, respectively.

As discussed in section 1 , the existing actuator surface model cannot be directly applied to the propeller blade because 2-D elements cannot be easily constructed and the required force coefficient may not be available.

The key idea of the proposed actuator surface model is to compute the forces on the actuator surface using RANS simulations. Specifically, the force on the actuator surface is computed by

$\boldsymbol{f}(\boldsymbol{X}, t)=\frac{1}{2} U^{2}(t) \cdot \boldsymbol{c}(\boldsymbol{X})$,

where $U(t)$ is the incoming velocity which may vary in time, and $\boldsymbol{c}(\boldsymbol{X})$ represents the force coefficients, which are calculated using the forces from the RANS simulation as follows:

$\boldsymbol{c}(\boldsymbol{X})=\frac{\boldsymbol{f}^{\text {RANS }}(\boldsymbol{X})}{\frac{1}{2}\left(U^{\text {RANS }}\right)^{2}}$,

where $f^{\text {RANS }}(\boldsymbol{X})$ is the force on the blade surface calculated from the RANS simulation consisting of the pressure and the friction components written as follows:

$\boldsymbol{f}^{\mathrm{RANS}}(\boldsymbol{X})=\boldsymbol{f}_{p}^{\text {RANS }}(\boldsymbol{X})+\boldsymbol{f}_{\tau}^{\text {RANS }}(\boldsymbol{X})$, 


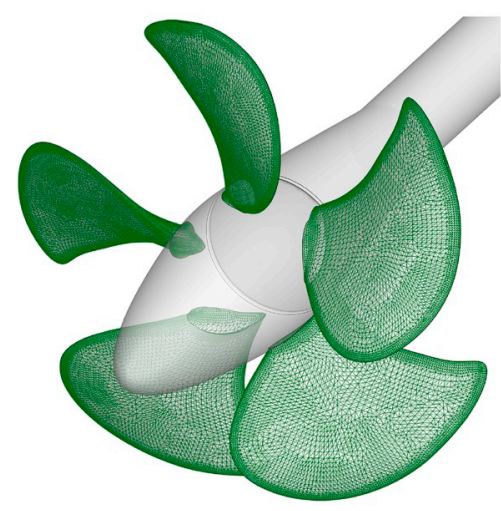

(a) Model M1

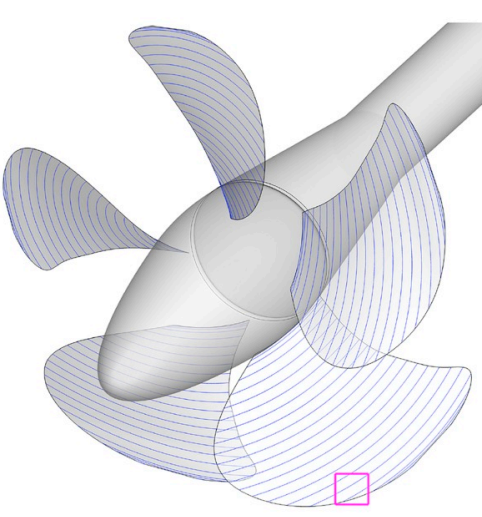

(b) Models M2 and M3

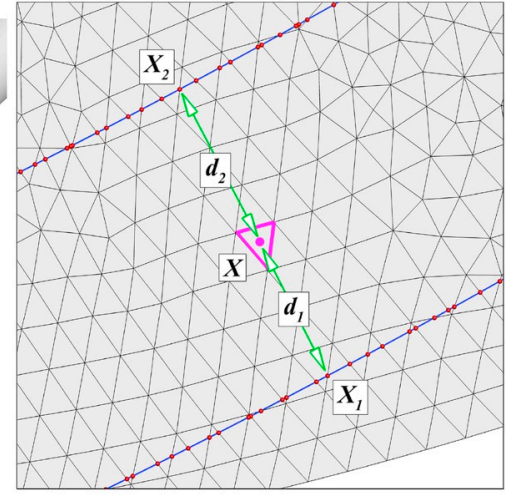

(c) Zoomed-in surface triangular mesh for M2 and M3

Fig. 3. Illustration of the blade geometrical representation of the three different actuator surface models for (a) model M1 and (b, c) models M2 and M3. Model M1 directly adopts the RANS mesh as the actuator surface, while models M2 and M3 employ a thickless actuator surface formed with curved lines as shown in (b). It is noted that the thickless actuator surface is still discretized using triangular elements as for model M1. The triangular surface mesh for the actuator surface is shown in (c). Only the RANS-computed forces on the curved lines shown in (b) are employed in models M2 and M3. The forces on the triangular cells located between two curved lines are interpolated using the inverse distance approach before the force distribution process (Eq. (4)).

where

$\boldsymbol{f}_{p}^{\mathrm{RANS}}(\boldsymbol{X})=\frac{1}{\rho}\left(p-p_{\infty}\right) \cdot \boldsymbol{n}$

$\boldsymbol{f}_{\tau}^{\mathrm{RANS}}(\boldsymbol{X})=-\nu \frac{\partial \boldsymbol{u}}{\partial n}$,

where $\boldsymbol{n}$ is the unit outward normal vector on the blade, the $p_{\infty}$ is the farfield pressure, the $\rho$ is the constant density, and the $\nu$ is the kinematic viscosity on the blade. It is noted that the forcing term $\boldsymbol{f}(\boldsymbol{X}, \boldsymbol{t})$ in Eq. (5) represents the force in the LES. Its time dependence comes from the incoming velocity $U(t)$, which may vary in time for complex inflow conditions. The force coefficients $\boldsymbol{c}(\boldsymbol{X})$, on the other hand, are assumed to be constant and computed from RANS simulations. In the present work, the Fluent software using the rotating reference frame technique, which divided the computational domain into the rotating domain and stationary domain and solves different forms of Navier-Stokes equation in different domains, is employed for the RANS simulation. It is noted that the surface forces computed from the RANS simulation need to be transformed using the rotation matrix before they can be employed to compute the force coefficients using Eq. (6) because of the use of the rotating reference frame technique.

To apply the RANS-computed forces to LES of the propeller wake, three different actuator surface simplifications, namely M1, M2 and M3, are tested. In the model M1, the actual blade geometry is employed as shown in Fig. 2. In the LES for predicting the propeller wake, the RANScomputed forces are directly distributed from the actual blade geometry, which is discretized using the same mesh as in the RANS simulations. The model M1 is considered to be the most accurate among the three models.

In models M2 and M3, on the other hand, the blade thickness effect is intentionally taken away by replacing the original blades with thickless ones. To obtain the geometry of the thickless blade, the original thick blades are cut into a series of curved elements at different radial locations as shown in Fig. 3. Then, each element is replaced with its corresponding mean camber line. Finally, after connecting these camber lines together, the thickless blades can be obtained.

In models M2 and M3, only the forces on the curved lines as shown in Fig. 3 are employed in Eq. (4) to compute the forces on the background Cartesian grids, which are computed as follows:

$\boldsymbol{f}^{\mathrm{RANS}}\left(\boldsymbol{X}_{L}\right)=\boldsymbol{f}^{\mathrm{RANS}}\left(\boldsymbol{X}_{L}^{+}\right)+\boldsymbol{f}^{\mathrm{RANS}}\left(\boldsymbol{X}_{L}^{-}\right)$,

where $\boldsymbol{X}_{L}^{+}$and $\boldsymbol{X}_{L}^{-}$represent the pressure side and suction sides of the
Table 1

Three different actuator surface models for the rotating blades of marine propellers.

\begin{tabular}{lll}
\hline Model & Geometry & Force \\
\hline M1 & Actual geometry & Actual RANS force \\
M2 & Thickless surface & Force on 2-D elements \\
M3 & Thickless surface & Chordwise-averaged force on 2-D elements \\
\hline
\end{tabular}

airfoil, respectively. Since the positions of the grid nodes on the curved element are in general not coincident with the background nodes, interpolations using the inverse distance method is employed to calculate the RANS-computed forces on the curved element. The RANS forces calculated on the curved lines are then employed in Eq. (6) for the calculation of the force coefficients. In the model M3 the force coefficients computed for the model M2 are averaged in the chord-wise direction before they are employed in Eq. (5) to compute the forces on the background Cartesian grid nodes. It is noted that in this work the thickless actuator surface is still discretized using the triangular mesh, for which the positions of the grid nodes may not coincide with the grid nodes on the curved lines shown in Fig. 3 (b). In this case the forces on the triangular grid nodes are interpolated from the curved lines. The key features of the three different models are summarized in Table 1.

The models M2 and M3 are formulated following the ideas of the two actuator surface models of Shen et al. (2009) and Yang and Sotiropoulos (2018), respectively. Compared with the model M1, models M2 and M3 lose not only the geometry (i.e. blade thickness) information but also the information of the force distribution on the blade surface. In the following section we will evaluate the capability of the three models in predicting the wake in the downstream of the VP1304 propeller.

Table 2

Principal geometry of the VP1304 propeller.

\begin{tabular}{llll}
\hline Propeller diameter & $D$ & 250.0000 & {$[\mathrm{~mm}]$} \\
Number of blades & $\mathrm{z}$ & 5 & {$[-]$} \\
Mean pitch & $P_{\text {mean }}$ & 391.8812 & {$[\mathrm{~mm}]$} \\
Thickness at $\mathrm{r} / \mathrm{R}=0.75$ & $t_{0.75}$ & 3.7916 & {$[\mathrm{~mm}]$} \\
Pitch at $\mathrm{r} / \mathrm{R}=0.75$ & $P_{0.75}$ & 407.3804 & {$[\mathrm{~mm}]$} \\
Pitch at $\mathrm{r} / \mathrm{R}=0.70$ & $P_{0.70}$ & 408.7500 & {$[\mathrm{~mm}]$} \\
Chord length at $\mathrm{r} / \mathrm{R}=0.75$ & $c_{0.75}$ & 106.3476 & {$[\mathrm{~mm}]$} \\
Chord length at $\mathrm{r} / \mathrm{R}=0.70$ & $C_{0.70}$ & 104.1670 & {$[\mathrm{~mm}]$} \\
Hub/Propeller diameter ratio & $d_{h} / D$ & 0.15 & {$[-]$} \\
\hline
\end{tabular}




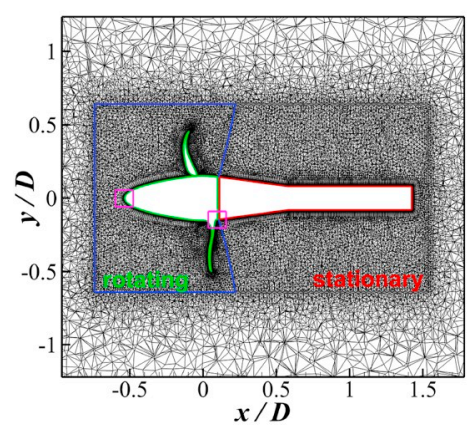

(a) Slice at $z=0$

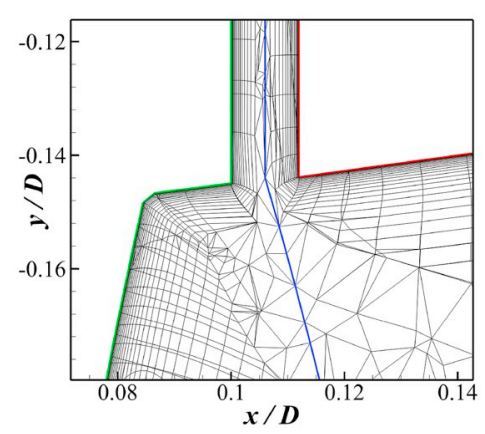

(b) Zoomed-in mesh around the gap

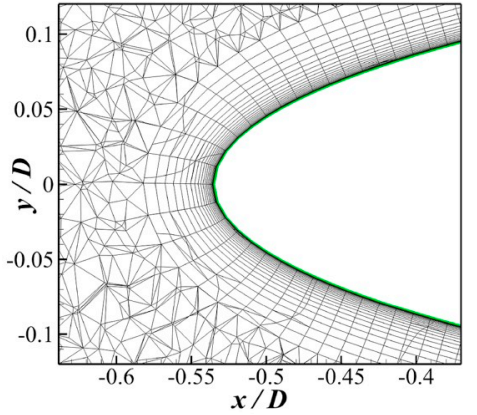

(c) Zoomed-in mesh around the front of the nacelle

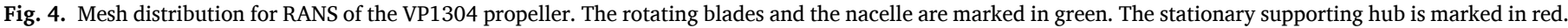

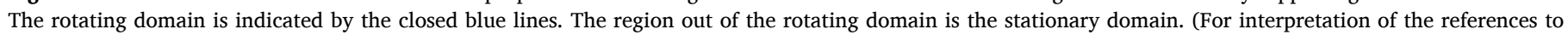
colour in this figure legend, the reader is referred to the Web version of this article.)

Table 3

Mesh distribution for steady RANS of the VP1304 propeller.

\begin{tabular}{llll}
\hline Geometry & Mesh type & Nodes & Elements \\
\hline Five blades & surface & 33807 & 67084 \\
Nose cap & surface & 7367 & 14220 \\
Supporting hub & surface & 8844 & 9692 \\
Rotating domain & volume & 1437669 & 3852983 \\
Stationary domain & volume & 669865 & 2670323 \\
\hline
\end{tabular}

Table 4

Principal parameters for propeller flows.

\begin{tabular}{lll}
\hline Advance coefficient & $J$ & $\frac{U}{n D}$ \\
Thrust coefficient & $K_{T}$ & $\frac{T}{\rho n^{2} D^{4}}$ \\
Torque coefficient & $K_{Q}$ & $\frac{Q}{\rho n^{2} D^{5}}$ \\
Open water efficiency & $\eta_{0}$ & $\frac{J}{2 \pi} \frac{K_{T}}{K_{Q}}$ \\
\hline
\end{tabular}

\section{Results}

In this section, we test the proposed actuator surface models for simulating the wake of the VP1304 propeller, which was provided by SVA Potsdam in the SMP'11 workshop at https://www.sva-potsdam.de/ en/pptc-smp11-workshop/. The geometry of the VP1304 propeller has been shown in Fig. 1 with geometric parameters displayed in Table 2. Incoming flow is in the $x$-direction. The origin of the $x$-axis is located at the pitch axis of the blades. The downstream location is based on the distance from the origin along the $x$-axis. In section 4.1, we first present the RANS results of the open water test of the VP1304 propeller. After that we present the LES results of the wake in the downstream of the VP1304 propeller using the three different actuator surface models M1, M2 and M3 in section 4.2.

\subsection{RANS simulation of the VP1304 propeller}

As mentioned in Section 2.2, RANS simulation of the VP1304 propeller is performed. Body-fitted boundary layer mesh is generated around the blades, nacelle and hub. For computational simplicity, the shaft which connects the rotating nacelle with the supporting hub in Fig. 1 is replaced with an empty gap in Fig. 4. Detailed mesh distribution is shown in Fig. 4 and Table 3. The $y^{+}$of the first cell on the wall in the boundary layer is about 1 . Steady convergence is achieved with the convergence residual going down by more than three orders of magnitude in 15 min for each simulation.

We employ the open water test results (Barkmann et al., 2011) for

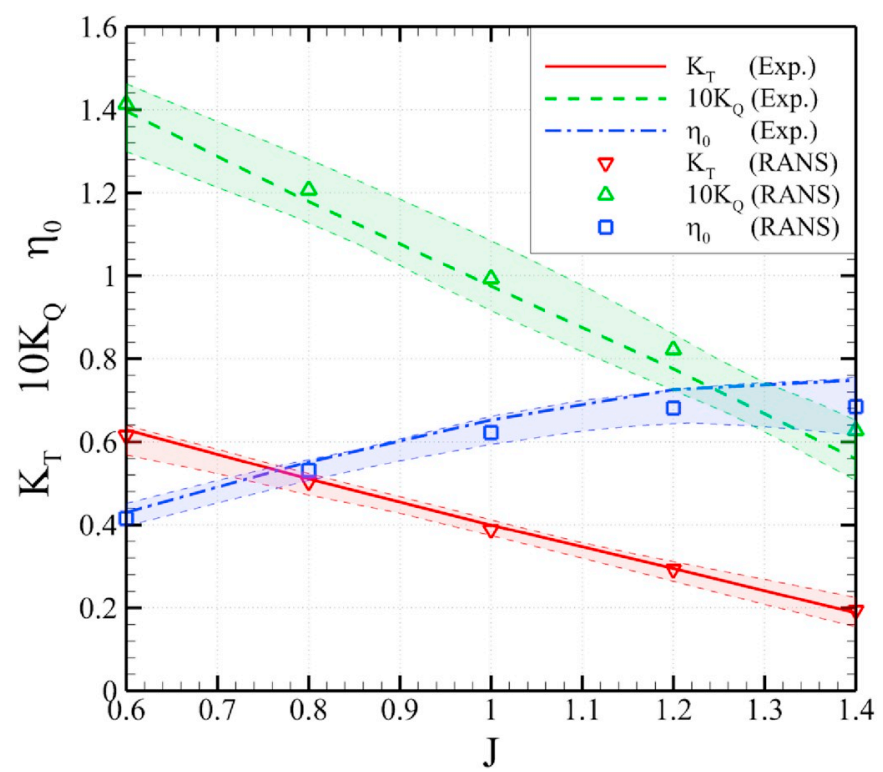

Fig. 5. Open water curves of the VP1304 propeller through RANS. The filled band regions indicate the computational results from the participants for the SMP'11 workshop.

validating the RANS results. Specifically, we compare the open water characteristics including the advance coefficient, the thrust coefficient, the torque coefficient, and the open water efficiency (defined in Table 4) computed from the RANS simulation with the measurements. Five incoming velocities, i.e. $2.25 \mathrm{~m} / \mathrm{s}, 3.00 \mathrm{~m} / \mathrm{s}, 3.75 \mathrm{~m} / \mathrm{s}, 4.50 \mathrm{~m} / \mathrm{s}$, and $5.25 \mathrm{~m} / \mathrm{s}$ corresponding to five different advance coefficient $J=0.6$, $0.8,1.0,1.2,1.4$, respectively, are considered. The rate of the rotor revolution is $n=15 \mathrm{~s}^{-1}$. The water density is $\rho=998.67 \mathrm{~kg} / \mathrm{m}^{3}$. The kinematic viscosity of water is $\nu=1.070 \times 10^{-6} \mathrm{~m}^{2} / \mathrm{s}$. The water temperature is $17.5^{\circ} \mathrm{C}$.

It is noted that our open water curves are obtained by considering the thrust and torque only on the blades and the nacelle without the supporting hub. As shown in Fig. 5, the RANS results agree well with the experiment.

The pressure distributions on the blade surfaces are shown in Fig. 6 . As seen, a strong pressure gradient is observed on the leading edge and the tip of the blades. With the increase of the advance coefficient $J$, the pressure gradient at blades' tip becomes weak and unnoticeable till $J=$ 1.4 . 


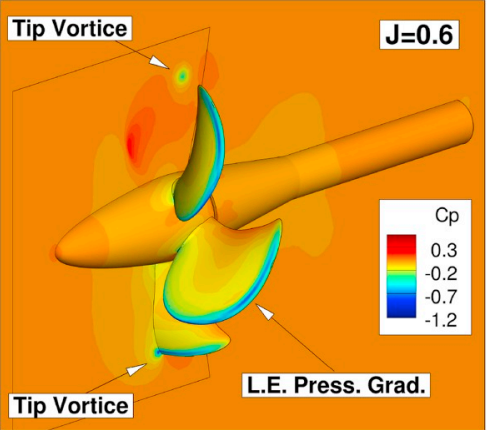

(a) $J=0.6$

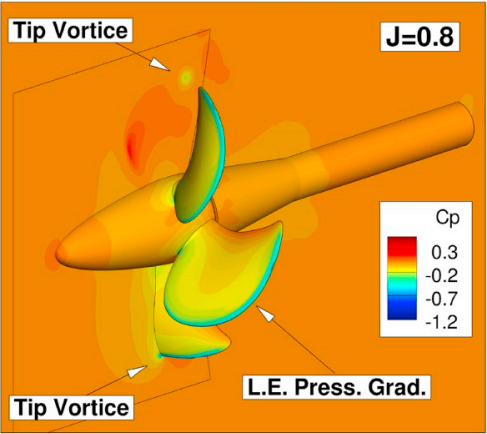

(b) $J=0.8$

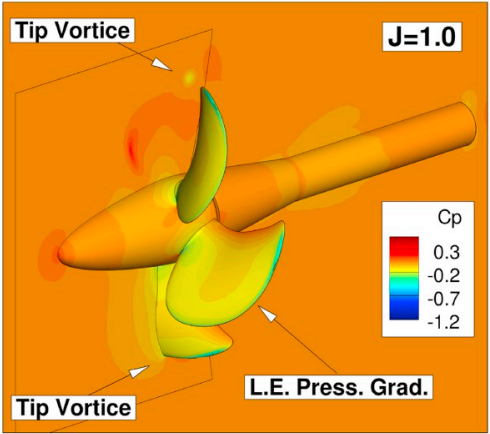

(c) $J=1.0$

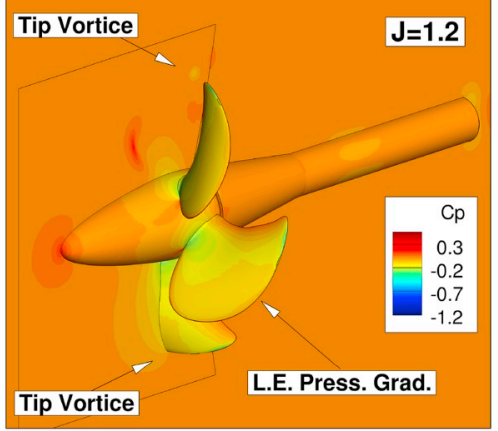

(d) $J=1.2$

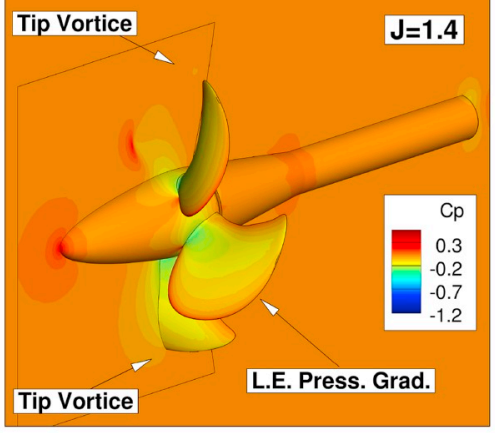

(e) $J=1.4$

Fig. 6. RANS-computed pressure distribution on the surface of the VP1304 propeller for different advance coefficients. Tip vortex and large pressure gradient at leading edge (L.E.) can be observed.

u/U: $\quad-0.22 \quad 0.1 \quad 0.42 \quad 0.74 \quad 1.06 \quad 1.38 \quad 1.7$

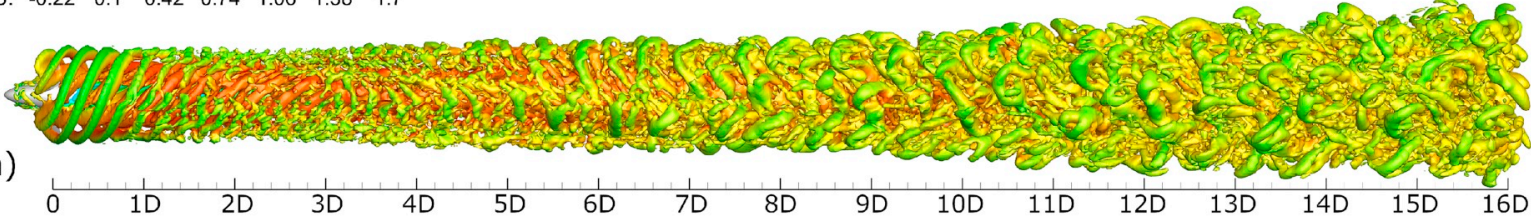

(b)

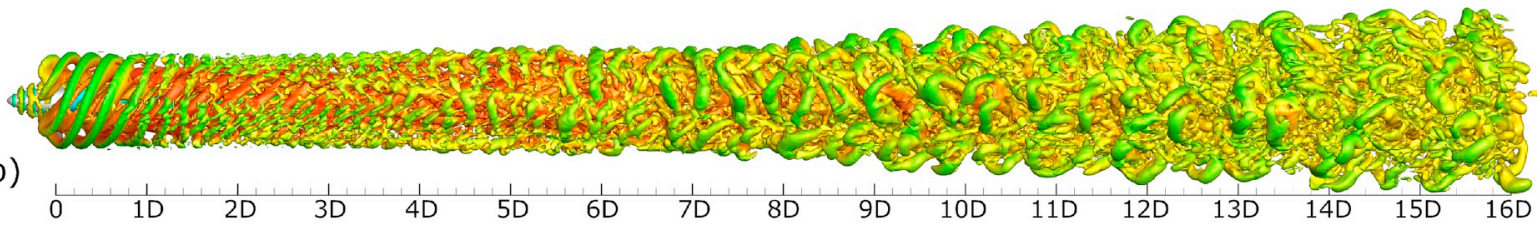

(c)

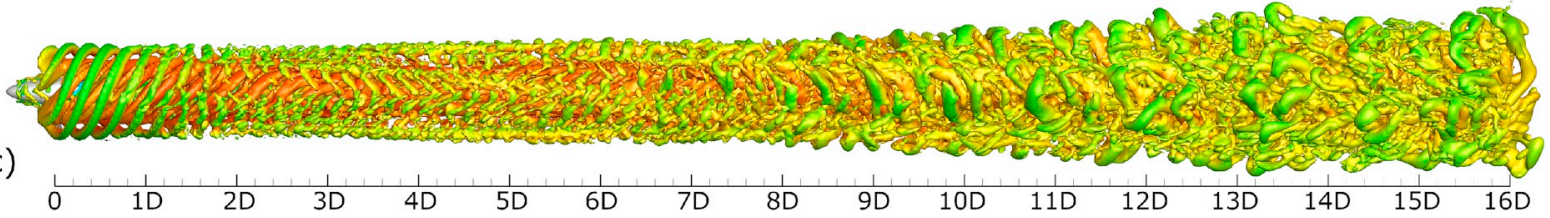

Fig. 7. Coherent flow structures in the downstream of the propeller identified using the Q-criterion computed using LES with three different actuator surface models for (a) M1, (b) M2 and (c) M3, respectively.

\subsection{LES of the VP1304 propeller using three different actuator surface models}

In this section, we present the large-eddy simulation results for the three different actuator surface models, where the distributed forces are computed from the separate RANS simulation as described in section 4.1. In LES, we only consider the advance coefficient $J=1.0$ with the incoming velocity $U=3.75 \mathrm{~m} / \mathrm{s}$. The dimension of the computational domain is $L_{x} \times L_{y} \times L_{z}=19.2 D \times 3.2 D \times 3.2 D$, where $D=0.25 \mathrm{~m}$ is the diameter of the propeller and $x, y$ and $z$ denote the streamwise, spanwise and vertical directions, respectively as in the RANS simulation. The total number of the background grid cells is $N_{x} \times N_{y} \times N_{z}=720 \times 120 \times$ $120^{\sim} 10$ million with the mesh demonstrated in Fig. 2(b). As seen the LES mesh is significantly coarser than the mesh employed in the RANS simulation near the propeller blade as there is no need to resolve the boundary layer over the blade in actuator surface models. The simulation is unsteady with the blades and nacelle rotating around the $x$-axis with the fixed speed of 15 round/s. The unsteady time step is set 


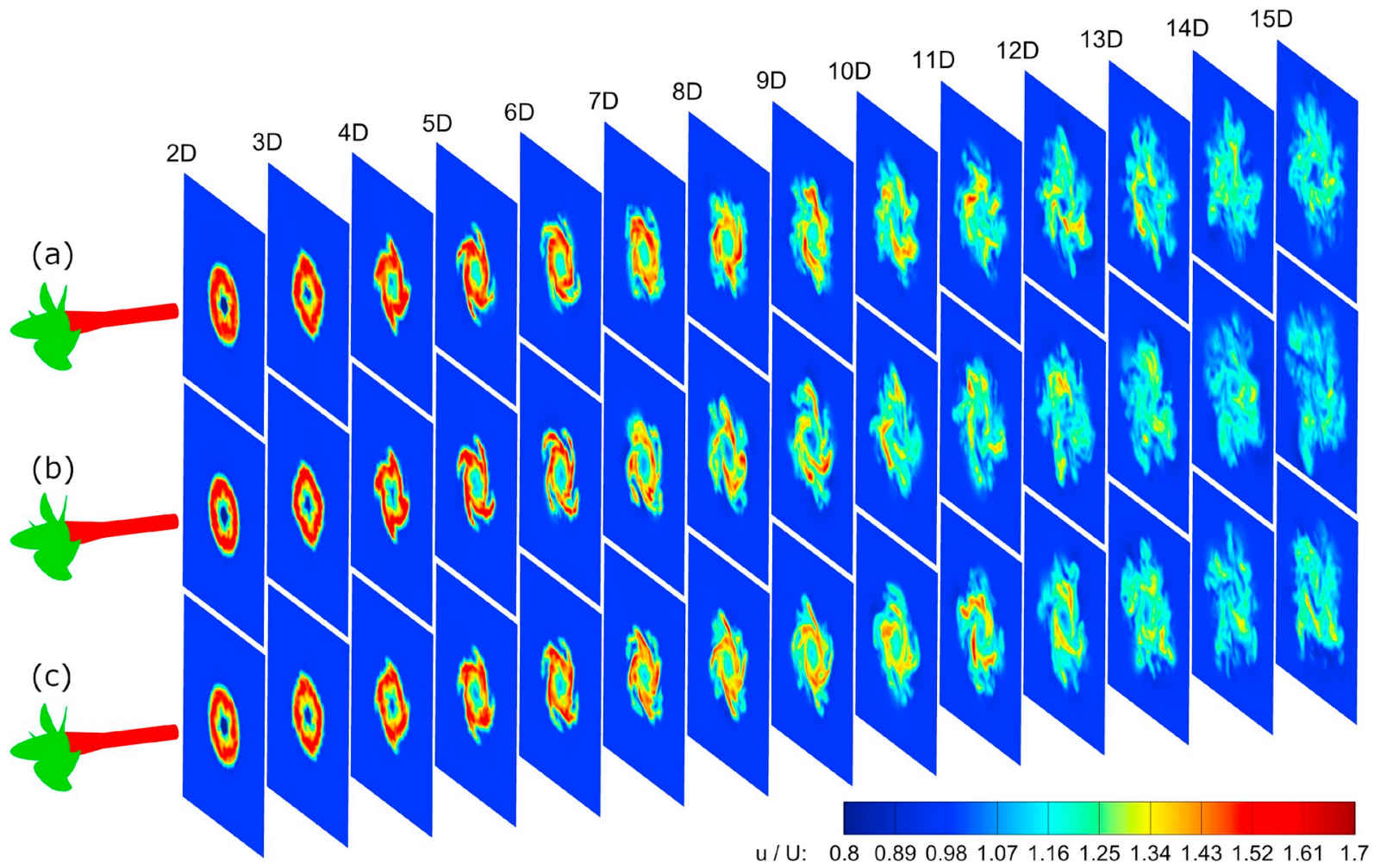

Fig. 8. Contours of the instantaneous streamwise velocity $u / U$ computed using LES with the three different actuator surface models for (a) M1, (b) M2 and (c) M3, respectively, at different downstream locations.

(a)

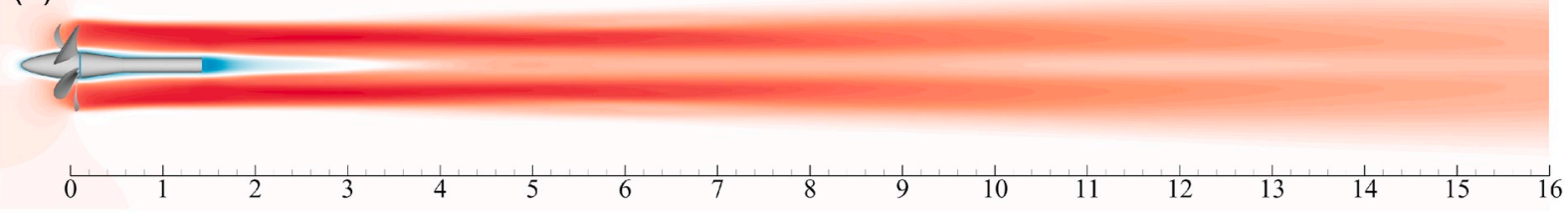

(b)

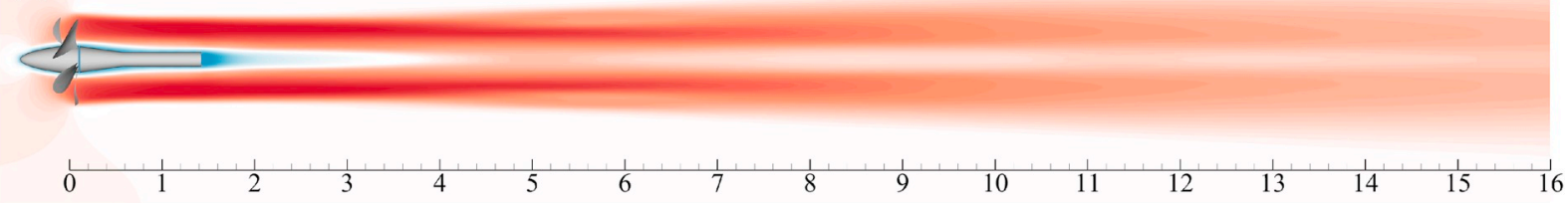

(c)

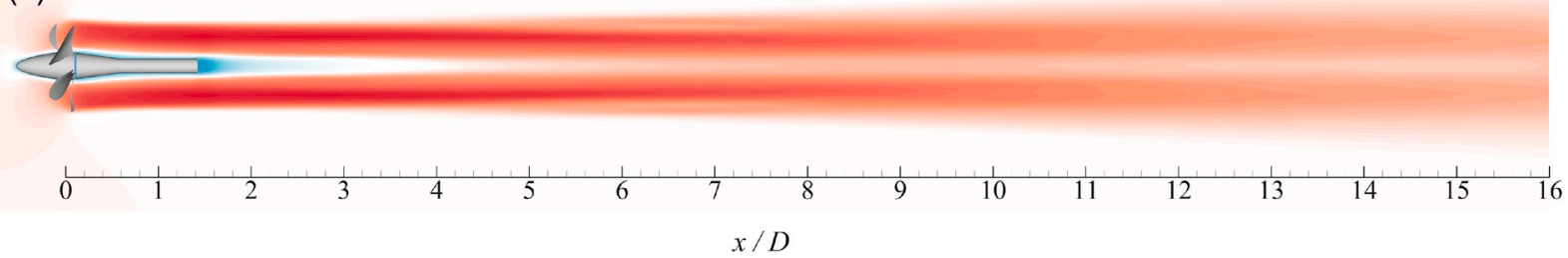

Fig. 9. Contours of the time- and azimuthal-averaged streamwise velocity $\langle u\rangle / U$ computed by LES using the three different actuator surface models for (a) M1, (b) M2 and (c) M3, respectively.

according to the maximum allowable Courant number 0.2 , which indicates that it takes about $5 \sim 6$ time steps for the blades' tips to pass one grid cell. Free-slip boundary condition is specified at the vertical boundaries and the spanwise boundaries. Uniform flow is specified at the inlet. Artificial damping is applied near the outlet of the computational domain to remove unphysical structures reflected from the outlet boundary.

First we examine the instantaneous iso-surfaces identified with the Q-criterion $Q=10$ as shown in Fig. 7 for the three different actuator surface models M1, M2 and M3. It can be seen that the tip vortex of cylindrical helix shape are well predicted by all three models at axial locations before $1 D$ downtream of the propeller. After $1 D$ downstream of 
(a)

$\begin{array}{lllllll}\langle v\rangle / U: & -0.45 & -0.27 & -0.09 & 0.09 & 0.27 & 0.45\end{array}$

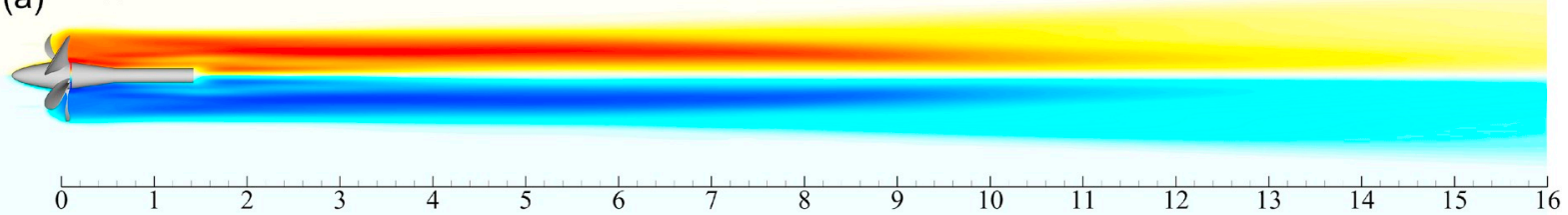

(b)

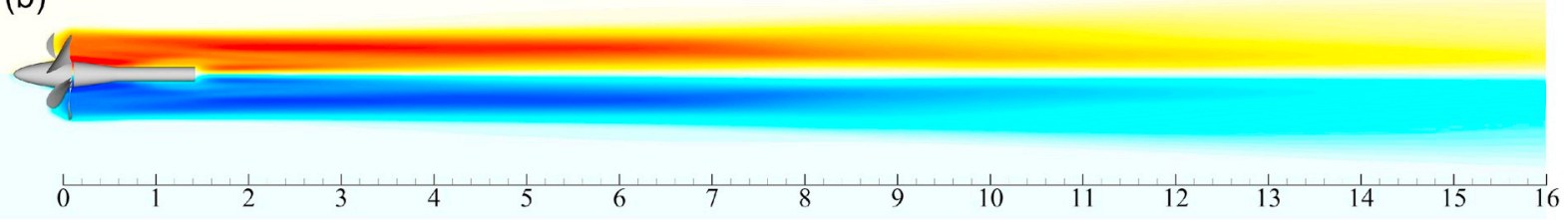

(c)

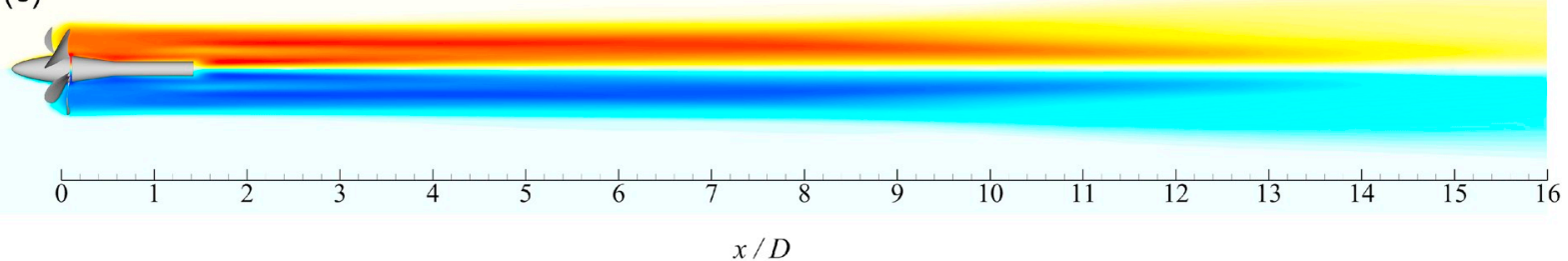

Fig. 10. Contours of the time- and azimuthal-averaged azimuthal velocity $\langle v\rangle / U$ computed by LES using the three different actuator surface models for (a) M1, (b) M2 and (c) M3, respectively.

(a) $k / U^{2}: \quad 0 \quad 0.0040 .0080 .0120 .016 \quad 0.02$

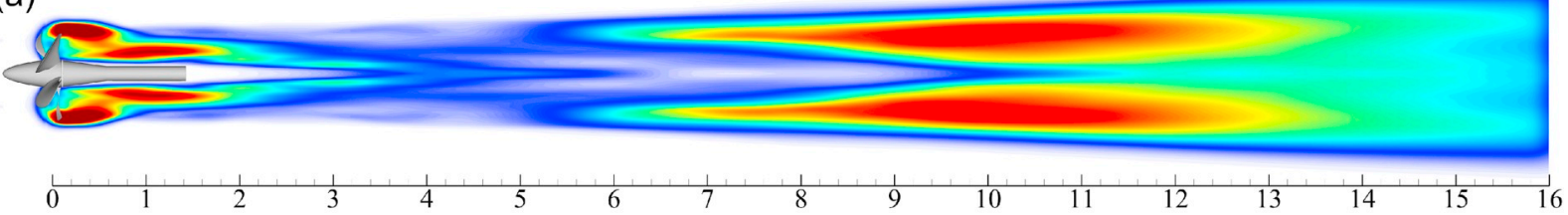

(b)

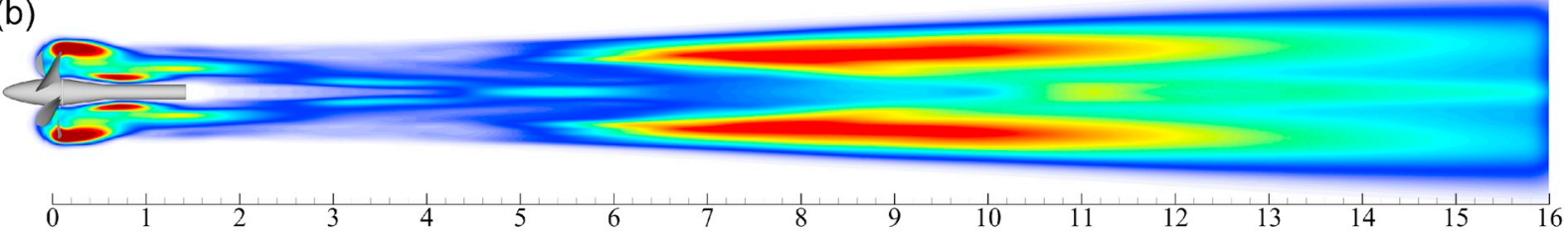

(c)

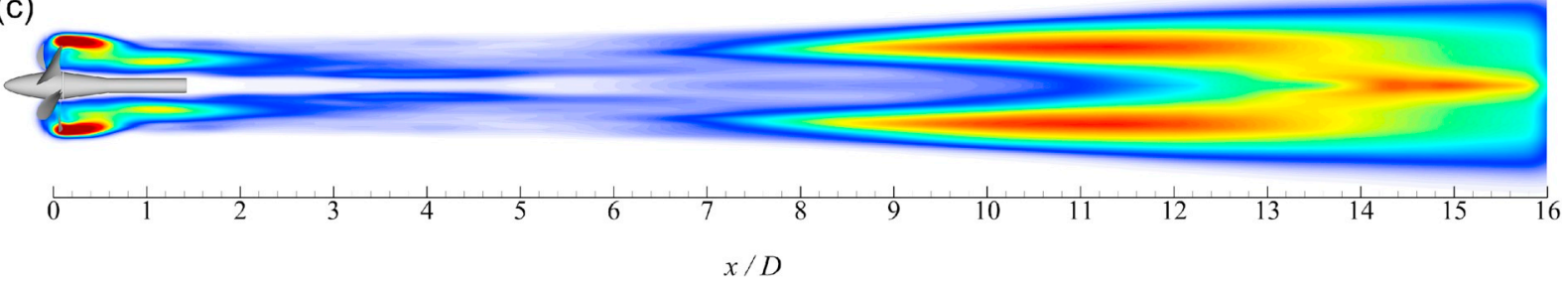

Fig. 11. Contours of the turbulence kinetic energy (TKE) $k$ computed by LES using the three different actuator surface models for (a) M1, (b) M2 and (c) M3, respectively. The computed TKE is further averaged in the azimuthal direction to plot the contours shown in this figure.

the propeller, the tip vortex computed using models M1 and M2 start breaking down into vortex segments, which happens slightly later for model M3. At further downstream locations of the propeller, large coherent structures start developing in the streamwise direction, which happens at $4 D$ propeller downstream for models M1 and M2 while $7 D$ propeller downstream for model M3.

Here we examine the contours of the instantaneous streamwise velocity $u / U$ at slices located at different downstream locations as shown in Fig. 8. As seen, the contour of concentric shape is observed at $2 D$ downstream of the propeller with the outer jet generated by the propeller and the inner wakes caused by the hub. At $4 D$ downstream of the propeller, four rotating tails appear, which gradually grow and break into small coherent structures at further downstream locations. The observations from the three models M1, M2 and M3 are similar with the rotating tail structure computed using the model M1 persisting slightly longer in the streamwise direction.

The contours of the time- and azimuthal-averaged streamwise velocity $\langle u\rangle / U$ computed by LES with the proposed three ASMs are shown 


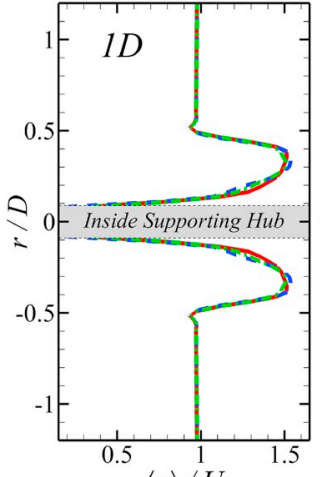

$\langle u\rangle / U$

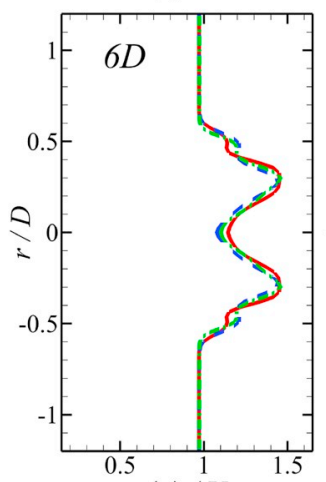

$\langle u\rangle / U$

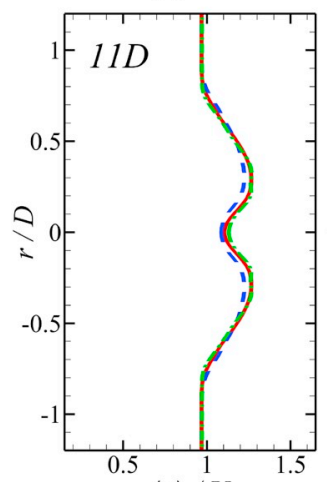

$\langle u\rangle / U$

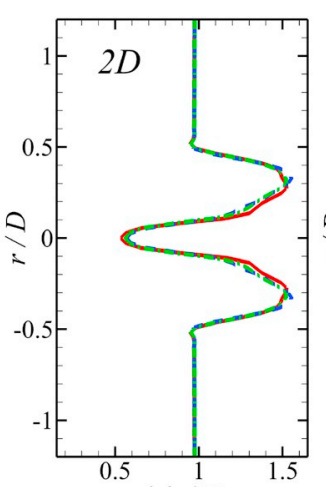

$\langle u\rangle / U$

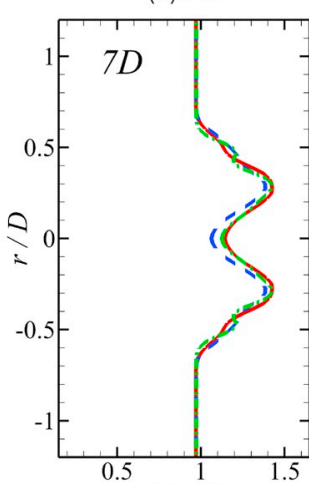

$\langle u\rangle / U$

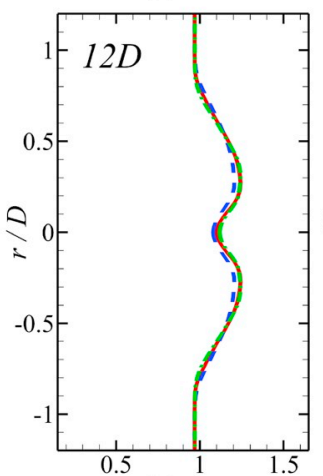

$\langle u\rangle / U$

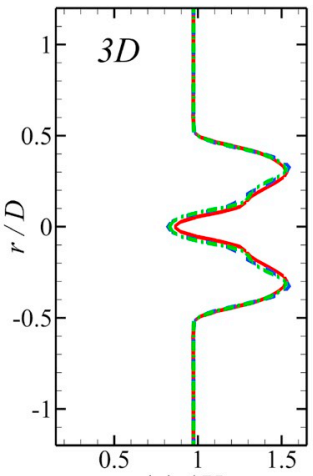

$\langle u\rangle / U$

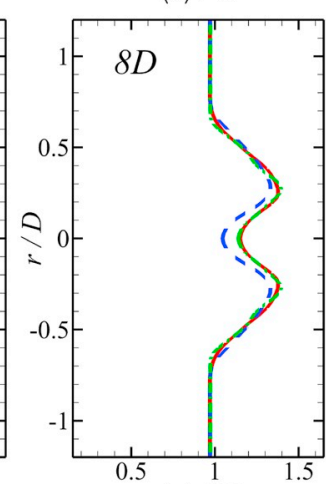

$\langle u\rangle / U$

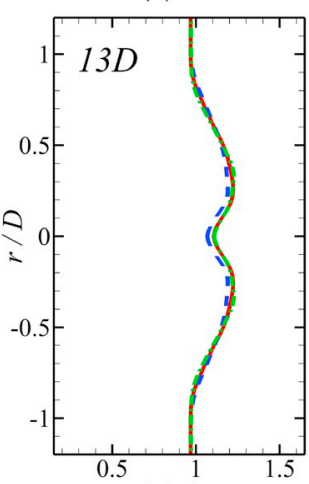

$\langle u\rangle / U$
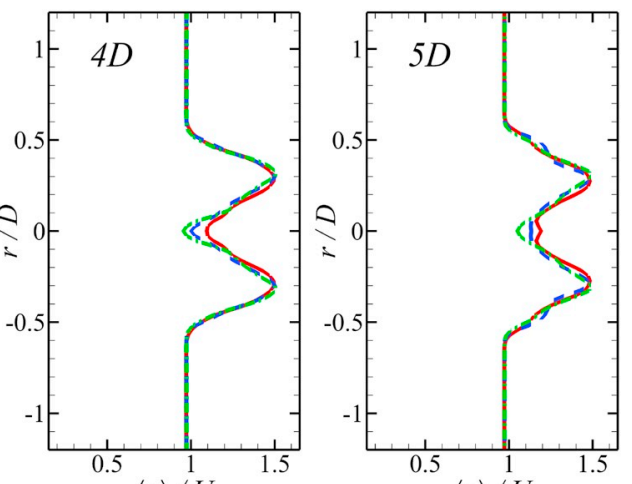

$\langle u\rangle / U$
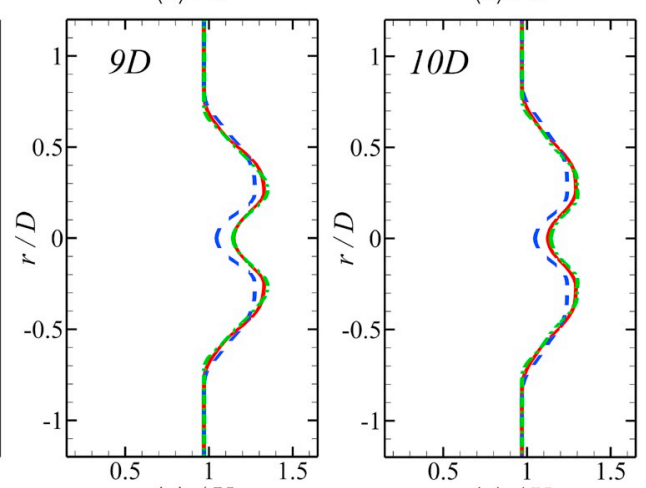

$\langle u\rangle / U$

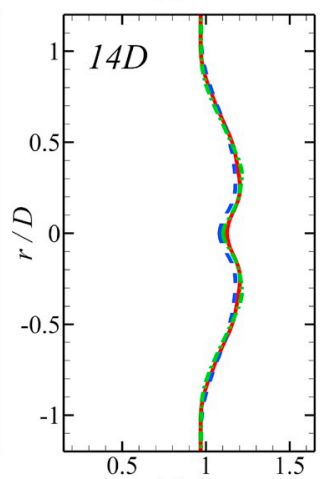

$\langle u\rangle / U$

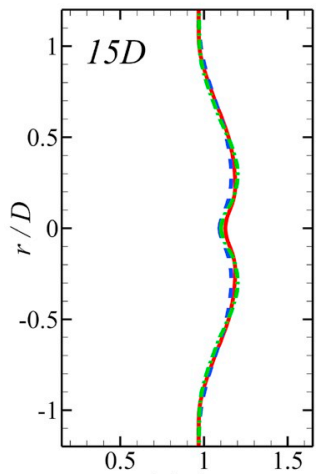

$\langle u\rangle / U$

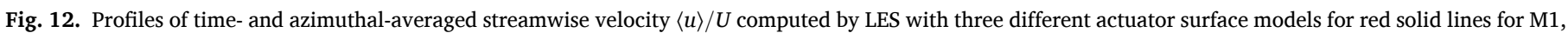

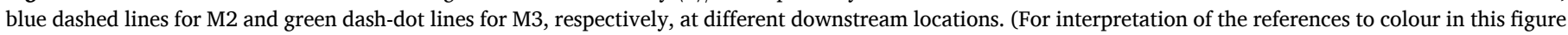
legend, the reader is referred to the Web version of this article.)

in Fig. 9. It can be observed that the model M1 produces slightly larger streamwise velocity among the three models. Detailed comparisons of the averaged streamwise velocity predicted using the three ASMs can be found in Fig. 12. The contours of the time- and azimuthal-averaged azimuthal velocity $\langle v\rangle / U$ computed by LES with the proposed three ASMs are shown in Fig. 10. All three models produce similar flow patterns. But the azimuthal velocity is observed to decay faster for model M1 compared with the other two models.

In Fig. 7 and 8, the coherent structures are observed evolving differently in the streamwise direction. Here we examine how these differences affect the distribution of the turbulence kinetic energy (TKE) $k / U^{2}$ in Fig. 11 . As seen the TKE intensity computed using the model M1 is larger than that computed using the models M2 and M3 at downstream locations $\left(0^{\sim} 3 D\right)$ close to the propeller. At further downstream locations two patches along the top and bottom tips of the propeller are observed for all the three models. However, the starting locations and the shape of the high TKE region are significantly different for the three models. The high TKE regions start at around 6D, 5D and 7D downstream of the propeller for the models M1, M2 and M3, respectively. As for the shape of the high TKE region, it is observed to be wider from 8D to $12 \mathrm{D}$ propeller downstream for the model M1. At further propeller downstream location $(>12 \mathrm{D})$ the TKE is observed to be weaker and homogenized in the spanwise direction for the models M1 and M2, but persistent strong and converging to the inner region of the wakes for the model M3.

We have qualitatively examined the differences across the three different actuator surface models. We now quantitatively examine the time- and azimuthal-averaged profiles of $\langle u\rangle / U$ and TKE at the 15 different propeller downstream locations in Fig. 12 and 13, respectively. Overall, the mean velocity profiles are observed being very similar for the three different actuator surface models. Relatively large differences are observed from 7D to 10D downstream of the propeller, where the streamwise velocity computed using the model M2 is about $10 \%$ lower than that computed using the models M1 and M3. Compared with the mean velocity profiles the differences in TKE profiles are observed to be larger for the three different models as shown in Fig. 13. At 1D propeller downstream the maximal TKE computed using the model M1 is approximately two times larger than the other two models. At 7D 

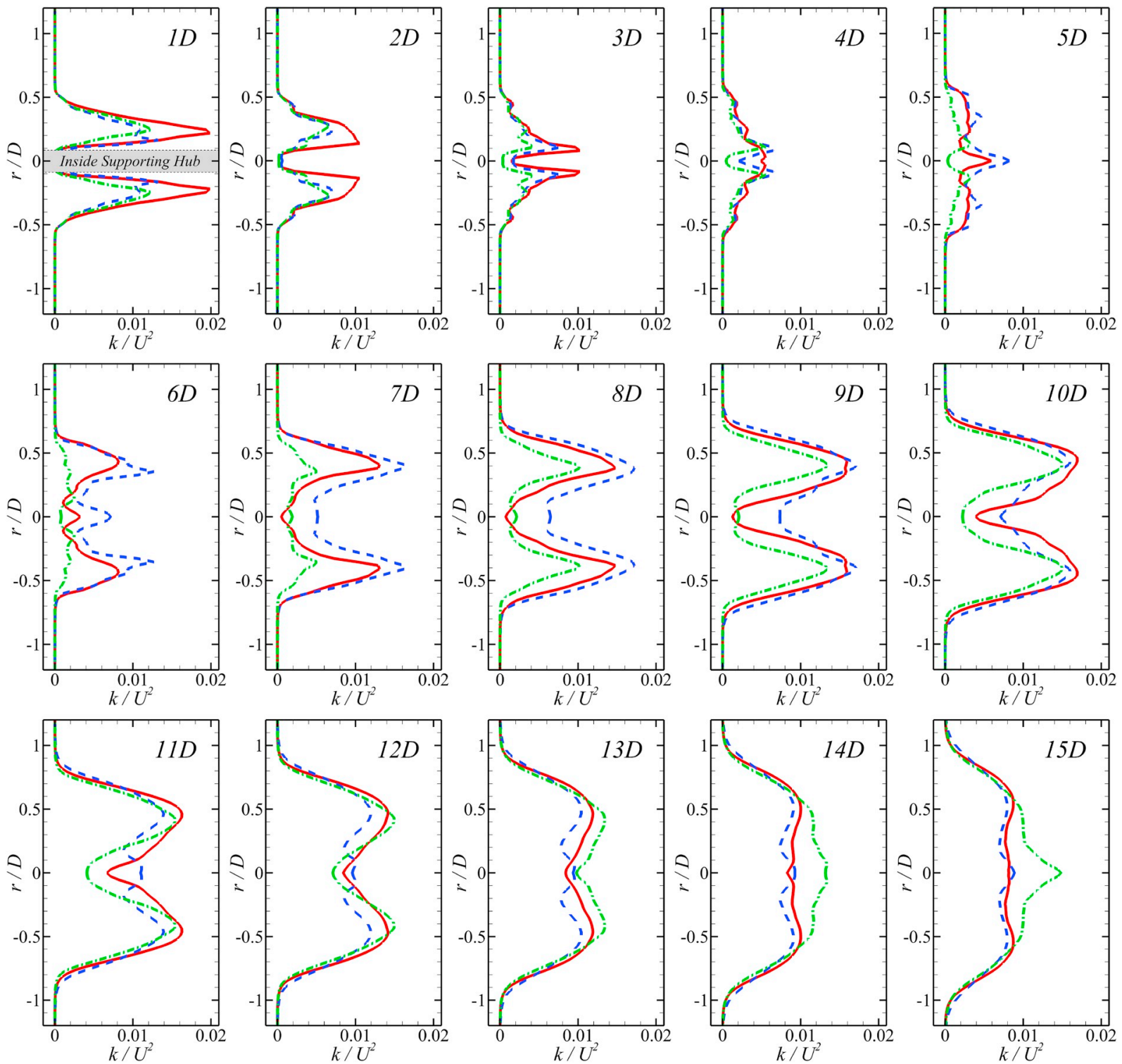

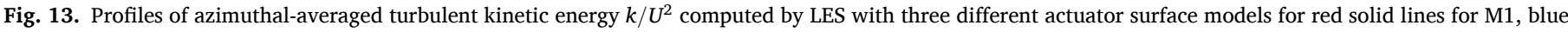

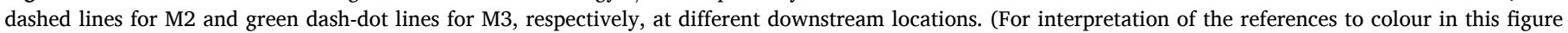
legend, the reader is referred to the Web version of this article.)

propeller downstream the maximal TKE computed using the model M3 is approximately only $30 \%$ of that computed using the other two models.

\section{Conclusion}

In this work we proposed a simulation-based actuator surface model with the distributed forces computed using the RANS simulation, the proposed actuator surface model, for marine propeller, for which the conventional actuator line/surface models for wind turbine blades can not be directly applied. We evaluated three different actuator surface models with different simplifications. For the model M1, the RANS computed forces are directly distributed from the blade geometry, which is discretized using the same mesh as in the RANS simulation. For the models M2 and M3, on the other hand, the forces are distributed from 2D elements (the force variation in the radial direction between two neighboring elements is neglected) of an actuator surface representation of the blade. The differences between models M2 and M3 is that the distributed forces are averaged in the chordwise direction in model M3. We evaluated the proposed actuator surface models by simulating the wakes downstream of the VP1304 propeller. The RANS simulation, which is employed for computing the distributed forces on the actuator surface, was validated using the data from the open water test. We compared the coherent structures, the mean velocity profiles and the TKE profiles computed using the three different actuator surface models. The differences in the mean velocity profiles are observed to be small for the three different actuator surface models. The differences in the TKE, on the other hand, are observed to be large especially between the model M3 and the other two models in terms of both the location and shape of the high TKE region. This indicates that the chordwise force distribution (which is assumed to be uniformly distributed in the model M3) has a significant effect on the TKE prediction in the downstream of the propeller, and has to be modeled properly in the actuator surface models for propeller wakes.

The present work focus on the wake prediction of marine propeller. However, it should be noted that the developed method can be directly applied to simulate the flow over wind and hydrokinetic turbines. Due to lack of experimental data especially in the far wake region, the improvement of the proposed model over the conventional ASM has not 
been quantitatively assessed in this paper. In the future work, a systematic evaluation of the proposed models will be carried out using the data from high fidelity geometry-resolved simulations and for different propellers.

One last note is that the proposed simulation-based actuator surface parameterization provides an approach for better taking into account the effect of the flow at small scales (e.g., the boundary layer over a blade) in large scale flow simulations (e.g., propeller wakes) in addition to the subgrid-scale model. In the present work, the flow over the blade is simulated using a RANS simulation. However, high-fidelity models like LES can also be employed in the small-scale simulations for situations when the RANS method is not very accurate (e.g., flows with massively separations). Furthermore, the simulation-based parameterization approach has the potential to be employed for modeling unresolved scales in large-eddy simulation of other large-scale turbulent flow systems, which will be pursued in out future work.

\section{Declaration of competing interest}

The authors declare that they have no known competing financial interests or personal relationships that could have appeared to influence the work reported in this paper.

\section{CRediT authorship contribution statement}

Fei Liao: Writing - original draft, Visualization, Formal analysis. Shizhao Wang: Conceptualization, Software, Resources, Methodology. Xiaolei Yang: Conceptualization, Methodology, Validation, Writing review \& editing. Guowei He: Supervision, Conceptualization, Project administration, Funding acquisition.

\section{Acknowledgment}

This work is supported by the NSFC Basic Science Center Program for 'Multiscale Problems in Nonlinear Mechanics' (No. 11988102), the National Natural Science Foundation of China (Nos. 11572331 and 91752118), and the National Numerical Wind Tunnel Project of China (NNW2018-ZT1A01). The authors would like to acknowledge the support from the Strategic Priority Research Program of the Chinese Academy of Sciences (XDB22040104) and the Key Research Program of Frontier Sciences of the Chinese Academy of Sciences (QYZDJ-SSWSYS002). The computations are conducted on Tianhe- 1 at the National Supercomputer Center in Tianjin.

\section{References}

Barkmann, Ulf, Heinke, Hans-Jürgen, Lübke, Lars, 2011. Potsdam propeller test case (PPTC). In: 14th Numerical Towing Tank Symposium, pp. 36-38.

Choi, Jung-Eun, Kim, Jung-Hun, Lee, Hong-Gi, Choi, Bong-Jun, Lee, Dong-Hyun, 2009. Computational predictions of ship-speed performance. J. Mar. Sci. Technol. 14 (3), 322-333.
Da-Qing, L.I., 2006. Validation of rans predictions of open water performance of a highly skewed propeller with experiments. Journal of Hydrodynamics, Ser. B 18 (3), 520-528.

Dobrev, I., Massouh, F., Rapin, M., 2007. Actuator surface hybrid model. J. Phys. Conf. 75, 012019.

Elias, Balaras, Schroeder, Seth, Posa, Antonio, 2015. Large-eddy simulations of submarine propellers. J. Ship Res. 59 (4), 227-237.

Froude, R.E., 1889. On the part played in propulsion by difference in pressure. Transactions of the Institution of Naval Architects 30, 390-423.

Ganesh Rajagopalan, R., Klimas, Paul C., Rickerl, Ted L., 1990. Aerodynamic interference of vertical axis wind turbines. J. Propul. Power 6 (5), 645-653.

Kang, Seokkoo, Borazjani, Iman, Colby, Jonathan A., Sotiropoulos, Fotis, 2012. Numerical simulation of 3D flow past a real-life marine hydrokinetic turbine. Adv. Water Resour. 39, 33-43.

Kang, Seokkoo, Yang, Xiaolei, Sotiropoulos, Fotis, 2014. On the onset of wake meandering for an axial flow turbine in a turbulent open channel flow. J. Fluid Mech. 744, 376-403.

Kawamura, Takafumi, Miyata, Hideaki, Mashimo, Kohji, 1997. Numerical simulation of the flow about self-propelling tanker models. J. Mar. Sci. Technol. 2 (4), 245-256.

Kumar, Praveen, Mahesh, Krishnan, 2017. Large eddy simulation of propeller wake instabilities. J. Fluid Mech. 814, 361-396.

Martin, O.L., 2015. Hansen. Aerodynamics Of Wind Turbines, third ed. Routledge.

Martinez, Luis A., Meneveau, Charles, Stevens, Richard, 2016. Wind farm large-eddy simulations on very coarse grid resolutions using an actuator line model. In: 34th Wind Energy Symposium, p. 1261.

Menter, F.R., 1994. Two-equation eddy-viscosity turbulence models for engineering applications. AIAA J. 32 (8), 1598-1605.

Nicoud, F., Ducros, F., 1999. Subgrid-scale stress modelling based on the square of the velocity gradient tensor. Flow, Turbul. Combust. 62 (3), 183-200.

Peng, Hongxuan (Heather), Qiu, Wei, Ni, Shaoyu, 2013. Effect of turbulence models on RANS computation of propeller vortex flow. Ocean. Eng. 72, 304-317.

Posa, Antonio, Balaras, Elias, 2018. Large eddy simulation of an isolated vertical axis wind turbine. J. Wind Eng. Ind. Aerod. 172, 139-151.

Posa, Antonio, Broglia, Riccardo, Felli, Mario, Falchi, Massimo, Balaras, Elias, 2019b. Characterization of the wake of a submarine propeller via large-eddy simulation. Comput. Fluid 184, 138-152.

Posa, Antonio, Lippolis, Antonio, Balaras, Elias, 2015. Large-eddy simulation of a mixedflow pump at off-design conditions. J. Fluid Eng. 137 (10), 101302.

Posa, Antonio, Broglia, Riccardo, Balaras, Elias, 2019a. LES study of the wake features of a propeller in presence of an upstream rudder. Comput. Fluid 192, 104247.

Shen, Wen Zhong, Sørensen, Jens Nørkær, Mikkelsen, Robert, 2005. Tip loss correction for actuator/Navier-Stokes computations. J. Sol. Energy Eng. 127 (2), 209-213.

Shen, Wen Zhong, Zhang, Jian Hui, Sørensen, Jens Nørkær, 2009. The actuator surface model: a new Navier-Stokes based model for rotor computations. J. Sol. Energy Eng. 131 (1), 011002.

Sørensen, Jens Nørkær, Shen, Wen Zhong, 2002. Numerical modeling of wind turbine wakes. J. Fluid Eng. 124 (2), 393-399.

Stern, F., Kim, H.T., Patel, V.C., 1988. A viscous-flow approach to the computation of propeller-hull interaction. J. Ship Res. 32 (4).

Wang, Shizhao, Shi, Beiji, Li, Yuhang, He, Guowei, 2016. A large eddy simulation of flows around an underwater vehicle model using an immersed boundary method. Theor.Appl.Mech.Lett. 6 (6), 302-305.

Wu, Yu Ting, Porté-Agel, Fernando, 2011. Large-eddy simulation of wind-turbine wakes: evaluation of turbine parametrisations. Boundary-Layer Meteorol. 138 (3), 345-366.

Yang, Xiaolei, Sotiropoulos, Fotis, 2018. A new class of actuator surface models for wind turbines. Wind Energy 21 (5), 285-302.

Yang, Xiaolei, Zhang, Xing, Li, Zhilin, He, Guo-Wei, 2009. A smoothing technique for discrete delta functions with application to immersed boundary method in moving boundary simulations. J. Comput. Phys. 228 (20), 7821-7836.

Yang, Xiaolei, Kang, Seokkoo, Sotiropoulos, Fotis, 2012. Computational study and modeling of turbine spacing effects in infinite aligned wind farms. Phys. Fluids 24 (11), 115107.

Yang, Xiaolei, Sotiropoulos, Fotis, Conzemius, Robert J., Wachtler, John N., Strong, Mike B., 2015. Large-eddy simulation of turbulent flow past wind turbines/farms: the Virtual Wind Simulator (VWiS). Wind Energy 18 (12), 2025-2045. 